\title{
Le crime était presque parfait. Meurtres, colère et désir des dieux dans un panthéon du Sud de l'Inde (Karnataka)
}

The nearly perfect crime: Murders, anger and desire among the gods in a southern Indian pantheon (Karnataka)

Jackie Assayag

\section{OpenEdition Journals}

Édition électronique

URL : http://journals.openedition.org/span/1522

DOI : $10.4000 /$ span. 1522

ISSN : 2268-1558

Éditeur

École pratique des hautes études. Sciences humaines

\section{Édition imprimée}

Date de publication : 1 août 1996

Pagination : 145-174

ISSN : 0294-7080

Référence électronique

Jackie Assayag, "Le crime était presque parfait. Meurtres, colère et désir des dieux dans un panthéon du Sud de l'Inde (Karnataka) », Systèmes de pensée en Afrique noire [En ligne], 14 | 1996, mis en ligne le 17 avril 2014, consulté le 09 octobre 2020. URL : http://journals.openedition.org/span/1522 ; DOI : https://doi.org/10.4000/span.1522 


\section{LE CRIME ETAIT PRESQUE PARFAIT Meurtres, colère et désir des dieux dans un panthéon du Sud de l'Inde (Karnataka) ${ }^{1}$}

\section{par}

\section{Jackie Assayag}

* En résumé, on peut dire que le rectangle de l'écran doit être chargé d'émotions *,

A. Hitchkock ${ }^{2}$.

Vous êtes dans le sud de l'Inde. Plus exactement dans le nord de cet Etat du Karnataka où l'on parle le kannada et vénère la déesse régionale Yellamma-Renukā. Vous faites face à un de ces modestes sanctuaires, comme il y en a tant, qui bariolent le paysage indien. Un jeune desservant (püjāri), de basse caste Kallar, y accueille les dévots moyennant émoluments. Des femmes vous y ont précédé. Debouts, devant le sanctuaire, elles chantent la grandeur, la * puissance " (sakti) surtout de cette " Mère » (amma) du " tout » (ella) - grattant à l'aide d'un plectre le luth monocorde (caudike) traditionnellement associé à

${ }^{1}$ Ce texte a fait l'objet d'un exposé dans le cadre du Groupement de Recherches "Anthropologie comparée du champ religieux" dirigé par M. Detienne.

${ }^{2}$ La citation est extraite de l'ouvrage de F. Truffaut (1966:45).

Destins de meurtriers

Systèmes de pensée en Afrique noire, 14, 1996 
cette divinité. Marmottant des formules rituelles, elles balancent un berceau en bois suspendu à la toiture de l'édifice contenant une petite poupée en chiffon. Ainsi anticipent-elles l'objet du désir : Yellamma, ce parangon d'épouse et de mère idéale (mutṭaãide), comble celles qui viennent lui demander un enfant.

Si maintenant vous dirigez le regard vers l'intérieur du sanctuaire, vous voyez une série de pierres, apparemment informes et anarchiquement disposées. En scrutant l'obscurité, vous découvrez qu'elles sont alignées longitudinalement sur deux plans successifs. Au premier plan, une pierre, presque sphérique, symbolise la tête décapitée de Yellamma et une autre, de forme oblongue, représente la hache (paraśu) de son fils, Paraśurāma ; chacune repose sur un large galet, appelé simbi, du nom de ce coussinet que les femmes indiennes posent sur leur tête lorsqu'elles portent des charges ou des récipients. Quant aux autres pierres plates levées derrière, elles figurent respectivement, en partant de la gauche : le tronc, les mains, les jambes, le grand (koda) puis le petit récipient à eau (samkoda) de Yellamma. Ce sont les figurations lithiques du corps morcelé de Yellamma (et de ses attributs). Pour avoir surpris la baignade érotique d'un roi avec ses courtisanes, la déesse fut en effet mise en piece à la hache par son propre fils sur ordre de son père - incarnation locale du grand Yogin Siva. L'ascète ne transige décidément pas sur la question de la pureté. Aussi lorsque Jamadagni-Śiva constata la souillure de son épouse, il entra dans une telle colere que le monde bascula.

Cet autel donne à voir davantage que le mythe puranique bien connu qui mentionne la seule décapitation ${ }^{3}$. Comme si, l'impitoyable Paraśurāma avait accompagné la décollation d'un dépeçage analogue à celui du Purusa - l'Homme primordial dont le sacrifice est à l'origine de l'ordre cosmogonique, selon le célebre hymne védique $(X, 90)$. Mais cet autel évoque surtout celui de Satī, l'épouse de Rudra-Śiva, dont les morceaux du cadavre tombèrent sur la terre

3 A côté des multiples variantes mythologiques de la culture populaire, indiquons les références écrites et lettrées des épisodes légendaires de Renukā : Mahäbhãrata III, 115-117 et XII, 49 ; Agnipurāna V ; Brahmåndapurăna II, 21-58 ; Renukämahätmya dans le Sahyādrikhanda. 
(indienne) en fixant les principaux "sièges" (pitha) des cultes à la déesse ${ }^{4}$. Ce dont atteste encore aujourd'hui le « siège " du pelerinage (tirtha) de Saundatti sur lequel règne la Yellamma dite aux "sept vallées (Elu kollada). Edifié au sommet d'une colline à laquelle la déesse a donné son nom, son temple occupe le centre d'une excavation naturelle de roche de forme elliptique, d'environ huit cents metres. Dans sa « fosse » (dongara) s'étoilent quelque soixante-dix sanctuaires, importants ou parfois minuscules, à l'exemple de celui, modeste, que nous venons de décrire.

De ces sanctuaires, il ne sera guère ici question ${ }^{5}$. On se concentrera plutôt sur les personnes divines qui habitent ce cratère. Plus précisément, sur leur désir et leur colere à l'origine du dramatique " roman familial "6 qui attire chaque année à en ce lieu salvateur des dizaines de milliers de dévots, notamment lors des deux grandes fêtes de pleine lune, du veuvage (hostila hunnime) et du (re)mariage (davanda hunnime) de Yellamma (et de Jamadagni). Célébrations qu'orchestrent les «prêtres » (püjări), de caste Bānajiga et de secte Vìrasaiva, selon une liturgie minutieusement conduite dans le sanctuaire de Jamadagni pour les funérailles, dans celui de Yellamma

${ }^{4}$ La tentation est également grande d'évoquer le mythe brahmanique de Satī, l'épouse de Rudra-Śiva, et la fille de Daksa. En effet, ce dernier, né d'un pouce de Brahmā et considéré comme la source de la vie cosmique, fut décapité par les guerriers de Śiva pour avoir oublié d'inviter leur dieu à un sacrifice de Visnu ; dans l'impossibilité de retrouver son chef, on le * recapita * d'une tête de bouc. Révoltéc par l'outrage paternel commis envers son époux, Śatī se jeta dans le feu sacrificiel (de Śiva). L'analogie avec Yellamma est d'autant plus suggestive que Daksa est à la fois le père et le fils d'Aditī que les versions savantes donnent comme la mère de Renukā (O'Flaherty 1973 : 30-31). De surcroît, le récit ne s'arrête pas là mais se poursuit en une espèce de version féminine de l'hymne védique $(X, 90)$ du Géant primodial (Purusa) dépecé (Renou 1956 : 97).

5 Pour la description des principaux sites cultuels de Saundatti, on se reportera à notre ouvrage (Assayag, 1992a: Part. I, chap. II, III, IV).

6 L'expression n'est pas employée dans sa stricte acception freudienne, c'est-à-dire des * fantasmes par lesquels le sujet modifie imaginairement ses liens avec ses parents * (Laplanche \& Pontalis $1967: 427$ ), mais pour suggérer, plus simplement, les riches associations que suscite l'histoire du divin * triangle * familial chez les dévots de Saundatti. 
pour les épousailles ${ }^{7}$. On s'arrêtera ici sur les mythes, où il est d'abord question de transgression, de violence et de sang, pour laisser dans l'ombre les rites, essentiellement organisés autour d'une célébration matrimoniale idéale. Gardons cependant en mémoire que seul leur rapport non-spéculaire explique que beaucoup viennent à Saundatti chercher l'augure fécond d'une divinité (subhadevi) qui incarne à la fois les maux et leurs remèdes. Si on a choisi de privilégier les récits recueillis auprès des membres des groupes sociaux subalternes - basses castes et Intouchables - c'est dans la mesure où ils éclairent, par une série de déplacements, d'hybridations ou d'inventions, le mythe pan-indien de Renukā. Autant de mises en intrigue de crimes en série, presque parfaits, illustrant la congruence entre machinerie sacrificielle et narrativité.

\section{Les mythes de fondation}

Le topos est fréquent en Inde. C'est par un massacre que débute l'épopée de Yellamma. Après avoir débarrassé l'endroit des démons (asura, räksasa) qui l'infestaient, la divine « Mère » (Amma) décida de s'installer « là " - sur cette "limite " (yelle), comme l'exprime un récit jouant sur son nom (yelle-amma). Le carnage vaut comme acte de fondation des rituels et des mythes avec lesquels la déesse se confond désormais. Cette victoire inaugurale établit sa suprématie définitive sur le monde. Peut alors commencer le récit de sa vie :

L'Intouchable Yellamma fut mariée à l'ascète-renonçant Jamadagni a la condition qu'elle lui apporte quotidiennement de la Malaprabha, l'eau nécessaire à ses ablutions rituelles. Sa chasteté lui donnait le pouvoir de confectionner un récipient à partir du sable pris dans le lit de la rivière. Elle le portait sur la tête, avec un cobra en guise de coussin (simbi). Chaque jour, accompagnée de sa servante Mātangi, Yellamma rapportait ainsi le récipient d'eau pour son époux.

${ }^{7}$ Pour une présentation synthétique des rapports entre caste et secte, sur le cas des Bānajiga-Lin̄gāyat, on lira Assayag 1994. 
Les années passèrent. Les enfants mâles du couple devinrent adultes et eux-mêmes des ascètes.

Un jour, en accomplissant sa tâche quotidienne, Yellamma surprit les ébats érotiques du roi Kārttavīrya avec ses concubines qui se baignaient dans la rivière. Prise d'un vif désir en contemplant ce spectacle, elle en oublia l'heure... Ne parvenant pas à modeler le pot en limon, elle retourna à l'ermitage, tard, et sans eau.

Jamadagni - possédé par la colère - se mit en rage contre sa femme fautive. Tout en la couvrant d'insultes, il lui jeta une malédiction qui lui fit perdre son charme corporel (leucodermie ou lèpre). Répudiée, Yellamma fut chassée de l'ermitage. Ainsi commença une longue errance.

Devenue mendiante en forêt, elle rencontra deux saints hommes shivaïtes, Ekināth(a) et Jōgināth(a). Pris de compassion pour sa miserable condition, ils lui conseillerent de se baigner dans le Jōgula Bhāvi (ou Jōgigala Bhāvi, « le bassin des sages »), d'adorer Śiva avec toute la dévotion (bhakti) dont elle était capable, de collecter les aumônes (jog wa) chaque jour dans cinq maisons differentes afin de les partager avec les pauvres ; à cette fin ils lui donnèrent une sébile en vannerie (padalage).

Suivant ces conseils avec ferveur, Yellamma recouvra son charme corporel. Aussi décida-t-elle de retourner voir son mari afin qu'il la reprenne. Dépité de la voir absoute de sa malédiction, et saisi une nouvelle fois par la colere, Jamadagni ordonna aux quatre premiers de ses fils (mariés) présents à l'ermitage, Vasu, Viśvavasu, Karna, Bhattakarna, de décapiter leur mère.

Tous, successivement, refusèrent. Courroucé par leur désobéissance, Jamadagni les maudit à leur tour en les transformant en eunuques pour le restant de leurs jours ${ }^{8}$. Il appela alors son jeune fils (célibataire) le plus dévoué, Parasurāma, qui se consacrait à la méditation loin de l'ermitage dans l'Himalaya. Le cadet obtempéra et décapita sur l'instant Yellamma.

Pour le récompenser d'avoir exécuté l'ordre avec célérité, Jamadagni lui demanda de faire deux voux. Par le premier, Paraśurāma souhaita voir ramenés à la vie ses quatre frères ${ }^{9}$, par le second, il ressuscita sa mère en la " recapitant ".

${ }^{8}$ C'est justement de ces quatre fils castrés de Jamadagni que les travestis de la déesse, dits jogappa, se réclament.

9 Les différents lignages des "prêtres * (Bānajiga) de Yellamma proclament descendre de ces quatre fils, "resexués et mariés *. 
Le cycle mythologique de Saundatti se fonde sur une relation conjugale ambiguë, sur l'impossible alliage entre le pur et l'impur : un Brahmane ascète et renonçant est marié à une femme tenue pour Intouchable par une majorité de dévots. De surcroît, l'épouse succombe à la séduction (involontaire) d'un roi. Peu importe que sa "faute " soit assimilee à une simple convoitise, comme l'explicite la version lettrée, ou que l'adultère soit effectivement consommé, comme y insistent les récits populaires. Dans l'un et l'autre cas, il s'agit bien d'une transgression: pulsion scopique ou passion amoureuse qui appellent également l'immolation. Une transgression qui a pour fonction d'inscrire le mythe dans l'histoire. Si la totalité du récit s'articule sur cette violation du désir (käma), c'est justement parce qu'une telle violation fonde la rupture avec le monde divin (qui pourtant connaît bel et bien la violence et la transgression) en composant une histoire qui n'est plus seulement celle des dieux mais échoit désormais aux humains. L'histoire des hommes (kaliyuga) commença par une faillite divine et ne s'inventa qu'avec l'irruption d'un désir malheureux.

Le temps des dieux fut celui où Yellamma, chaque jour, remplissait son récipient d'eau. Elle accomplissait alors son devoir d'épouse, obéissante et fidèle, sans faillir. Le temps des hommes s'engendra d'un trouble érotique provoqué par un regard fasciné; en réalité, un passage à l'acte lésant le væu conjugal au dire des Intouchables. Le « désir» (kāma), fauteur de trouble et facteur de désordre, le désir du désir, pour l'exprimer plus exactement, est à l'origine du « roman familial » et du roman de l'Origine de Saundatti. A l'instar de Ratī la divinité hindoue du plaisir - l'exact inverse de ce prototype épique d'épouse qu'est Sìtā, l'Irréprochable -, Yellamma est en la circonstance pleinement déesse de l'amour. Les Intouchables la considèrent comme la patronne des femmes à la sexualité mercenaire et l'assimilent à une "prostituée " (sule) lors de la célébration de son veuvage, fête appelée « la pleine lune de la putain " (rande hunnime). Pourtant, seule une fraction de femmes qui lui sont consacrées sont effectivement des prostituées. De leur côté, les médias les confondent frauduleusement avec celles qu'on désignait autrefois comme les " servantes (ou esclaves) du dieu "(deva-dāst) (Assayag 1990). 
L'histoire de Yellamma ne s'arrête pas là. Le site sur lequel elle établit son temple, dit Rāmśringāpārvata, est celui où son époux installa son ermitage :

L'ásrama forestier de Jamadagni était sous le patronage du roi Kārttavīrya.

Apprenant du messager des dieux, Nārada, que l'ascète possédait la Vache céleste qui réalise tous les vœux (Kāmadhenu), le roi envieux prétexta les fatigues d'une expédition de chasse pour venir s'y reposer. Ne soupçonnant nullement ses méchantes intentions, Jamadagni l'accueillit avec générosité. Il pourvut notamment à l'entretien de son armée grâce à la fécondité illimitée de la Vache céleste. A la suite du refus de Jamadagni de céder le divin animal, le roi tenta par la force de s'en emparer avant son départ. Mais la vache glissa entre ses mains, et s'envola au paradis. Désappointé, prit de fureur, Kārttavīrya massacra Jamadagni en lui infligeant vingt et une blessures.

Cet épisode, qui nous fait assister au débordement des fonctions normalement assignées au roi, le représentant de la seconde classe (Ksatriya) dans la quadripartition des "ordres" (vama), amplifie une situation de crise socio-cosmique (adharma). Une crise qu'avait déjà dramatisé, au plan familial, le désir coupable de Yellamma. En cherchant à s'emparer de la vache qui symbolise le pouvoir du Brahmane, le roi n'assure plus son devoir de protection envers l'ascète. Surtout, en commettant le crime ultime de brahmanicide, en principe inexpiable, il rompt l'harmonique complémentarité des deux varna supérieurs. Parce qu'il fait fi et de la hiérarchie des espèces sociales (varna ou jâti) et de son obligation de patronnage, ce roi dévoyé qu'est Kărttavīrya renverse l'ordre du monde de façon démoniaque. Ce qu'il faut entendre au sens strict puisque tant son nom, son histoire que son lien avec la déesse (Māriyamman), évoquent le « dieu criminel et dévot démon" tamoul Kāttavarāyan (Ramanujan 1986 : 46 ; Biardeau $1989 \mathrm{~b}: 30$; Shulman $1989: 50$ ).

Heureusement cependant, les dieux, comme les hommes, sont organisés en famille où la vertu d'obéissance et la transmission du devoir fonctionnent : Jamadagni et Yellamma ont un fils. Certes, il médite loin de l'ermitage où vient de se dérouler le drame. Mais, 
comme il se déplace avec une vitesse qui annule le temps, Saundatti n'est qu'à quelques enjambées :

Désemparée par le meurtre de son époux, Yellamma appela Paraśurāma. Découvrant le corps meurtri de son père, il fut saisi par la colère et jura de le venger en massacrant tous les Kșatriya de l'univers. Dans sa rage meurtriere, il parcourut vingt et une fois la terre et décima rois et princes sans exception.

Seuls les épouses et les enfants en réchappèrent en venant se réfugier à l'ermitage où ils obtinrent la protection de Yellamma.

A la violence extrême du roi qui brouille les limites traditionnellement fixées aux fonctions de chacun, répond la non moins radicale vengeance du célibataire-ascète, doté des attributs du Kșatriya, qui extermine l'ordre des guerriers. Le motif répète la geste destructrice à laquelle Parasurāma paraît constamment associé. On se souvient en effet comment, sur ordre paternel, il décapita sa mère dont les actes ou les pensées avaient souille l'univers. A toute transgression de l'ordre - exprimée ici par une confusion des devoirs et des lignes de conduite - semble devoir systématiquement répondre un plus grand désordre. Un désordre total, puisque la terre ne saurait se passer de roi et les hommes du respect des normes matrimoniales. Le paradoxe n'est qu'apparent. D'une part, Paraśurāma épargne les enfants dont le mariage avec les femmes des Brahmanes engendrera une nouvelle race (purifiée) de Kșatriya. D'autre part, il ne fait qu'accomplir la fonction qui le destine à rétablir l'ordre socio-cosmique (dharma), fût-ce par des moyens violents qui semblent le dénaturer. Certes, en déviant de son rôle propre (svadharma) de Brahmane, il fait preuve d'un comportement d'exception. Mais en période d'extrême détresse (ápadharma), ce comportement est licite, requis même au regard de la dégradation du cosmos (kaliyuga). Aux situations scandaleuses, provoquées par un roi brahmanicide et une épouse infidele, répond l'intervention, apparemment déréglée mais en réalité normative, du Brahmane combattant. Derrière la causalité vengeresse se profile ainsi le rétablissement des fonctions de chacun dans un Ordre (dharma) restauré. C'est donc dans la logique (puranique) des interventions et "descentes" (avatāra) divines qu'il faut chercher le modèle de 
Parasurāma - modèle cosmologique que les travaux de M. Biardeau ont explicité $(1968 ; 1969 \mathrm{a} ; 1976)$.

Les massacres de Yellamma et de Jamadagni n'expriment donc que le désordre cosmique présent. La tragique destinée des dieux de Saundatti illustre un monde où l'épouse commet l'adultère, où le fils devient matricide qui plus est sur ordre paternel, où l'ascète souillé est massacré par son protecteur, où le Brahmane enfin se transforme en guerrier. Ce débordement de toutes les fonctions a pour effet de renverser les règles et de dénaturer les êtres - la désorganisation est totale. Pourtant le drame anticipe presque simultanément une restauration, mais à condition que l'expiation soit conduite jusqu'à ses plus extrêmes limites, c'est-à-dire au gigantesque massacre qui n'épargnera aucun des acteurs. Seule en effet une hécatombe sacrificielle de cette ampleur autorisera la reconstitution du triangle familial fondateur, synonyme de régénération du cosmos idéal où le fils et la mère reprennent leur place, tous deux dévotement soumis à leur gourou père et mari Śiva- Jamadagni. La fureur de l'intervention sanglante du fils ascète, cette "violence qui ne souille pas celui qui la commet ou la fait commettre ${ }^{10}$, purifie la terre de ses souillures. Qu'on reconnaisse dans cette violence meurtrière la transformation spécifiquement indienne d'un conflit cedipien, par lequel Paraśurāma gagne honneur et pouvoir - "ego ideal for Hindu men ", écrit R.P. Goldman (1978 : 364) -, ne doit pas faire oublier qu'il s'agit d'abord du mythe de la naissance d'un héros. Obéissant aveuglément au père agressif, l'enfant matricide se transforme en avatāra hérö̈que selon un modele qui est d'abord cosmique, répétons-le.

\section{Paraśurāma, le bâtard}

A Saundatti un temple est dédié à Paraśurāma. On est d'abord surpris de la distance qui le sépare de celui de Yellamma. Il n'y a

${ }^{10}$ Cf. Malamoud (1984: 43) dont l'article éclaire le problème de la vengeance dans les Epopées. 
pourtant là nul mystère. Aucun dévot n'en ignore la raison: les rapports entre le fils et la mere sont exécrables. Au point que Yellamma lui interdit de s'installer à sa proximité ! Pour quelle raison, questionnera-t-on ? Parasurāma est un bâtard !

Un premier récit, qui évoque l'histoire du dieu guerrier Kārttikeya - né du sperme refroidi de Siva qui féconda sa co-épouse Gañāa (O'Flaherty 1973 : 96-110) - fait de cet enfant exceptionnel le fruit de la semence d'un roi :

Alors que le roi Kārttavīrya se baignait dans la rivière Malaprabha, il fut soudain ravi par le captivant parfum qui parvint jusqu'a lui. Subjugué, il scruta la berge, pour bientôt apercevoir une femme puisant de l'eau. C'était Yellamma. Sa beauté le fascina tant qu'il éjacula involontairement dans la rivière.

Peu de temps après Yellamma se baigna et fut accidentellement fécondée par le sperme du roi. Elle enfanta un fils quelques mois plus tard, qu'elle appella Paraśurāma.

L'enfant, grandissant, ne cessait d'interroger sa mère sur le mystère de sa naissance. Il accompagnait ses harcelantes questions de la musique obsédante de son luth monocorde. Yellamma refusait toujours de répondre et se mettait souvent en colère. Excédé de son côté qu'on l'appelle sans cesse " le bâtard ", Parasurāma s'évertuait à insulter sa mère, la traitant notamment de « putain ". L'inimitié entre eux devint telle qu'ils durent se séparer à jamais.

Un second récit propose une autre explication surnaturelle de sa naissance : Parasurāma est le produit d'une fécondation capillaire.

Née d'un couple de Brahmanes qui vivait à Chandragiri, Yellamma était la première fille d'une famille qui comptait déjà sept garçons. Très jeune, elle se fit remarquer par son intense dévotion (bhakti) au dieu Śiva.

Un jour, elle se rendit à la rivière pour s'y baigner, comme elle en avait l'habitude. Arrivée sur la berge, elle toucha par inadvertance les cheveux d'un saint (muni) appelé Jāmuka. A la suite de ce geste, Yellamma se retrouva enceinte. Ses sept frères ne purent le tolérer. Aussi, pour répondre à l'affront fait à leur sœur, ils assassinèrent Jāmuka Muni.

Au moment de l'accouchement, les sept frères envoyèrent un homme travesti en femme tuer l'enfant dès sa naissance. Miraculeusement cependant il en réchappa et, contre toute attente, ce fut l'assassin qui décéda. Devant cet exploit, les frères 
réalisèrent l'extraordinaire pouvoir de la progéniture et se tinrent dorénavant à l'écart de leur sœur.

L'enfant reçu le nom de Paraśurāma. Durant sa jeunesse, les personnes qui le croisaient prirent l'habitude de se moquer de lui, de l'insulter, de le traiter de "bâtard ". Aussi questionnait-il sans relâche sa mère sur son origine. Lorsqu'à l'âge de douze ans il apprit la vérité, sa colère fut telle qu'il tenta à plusieurs reprises de la décapiter. N'y parvenant jamais, il réalisa finalement que Yellamma n'était pas une simple mortelle. Terrorisé, il s'enfuit loin d'elle.

Dans les deux récits, la naissance de Paraśurāma, résultat d'une fécondation accidentelle, signe une intervention surnaturelle. Et, à chaque fois, elle se produit à la faveur d'excreta : sperme et cheveux. Or ces substances - impures lorsqu'elles sont séparées du corps - se révèlent ici tellement "chaudes " et puissantes qu'elles réussissent à imprégner à distance une femme de manière différée. Le constat ne vaut sans doute pas seulement pour le Karnataka, les substances et les fluides corporels : cheveux, urine, salive, sang, sueur, larmes, sont fréquemment des métonymies de la semence, et donc sexuellement marqués et pourvus d'une valeur fécondante (Ramanujan 1983 : 239). A plus forte raison lorsqu'il s'agit de la semence d'un roi. Et plus paradoxalement dans le cas d'un ascète, bien que son imposante chevelure puisse acquérir une fonction identique tant s'y concentre de puissance méditative (tapas). Tandis que le roi incarne l'érotisme fécond du royaume, l'ascète symbolise la puissance bénéfique du renoncement. L'identité respective des deux pères involontaires de Parasurāma révèle celle, vacillante, de l'enfant : divinité trouble à la fois brahmane et guerrière, mais aussi vishnouïte et shivaïte, comme le rappelle A. Gail (1977: 220-229).

A ces deux figures contrastées de la fertilité mondaine (royale) et ultra-mondaine (de l'ascète) reviennent l'équivoque privilège de transformer Parasurāma en bâtard et, du même coup, de faire basculer Yellamma du côté des filles-mères, deux situations, on le sait en Inde, fort stigmatisées. Mais l'équivoque révèle en même temps la dimension divine et de la mère célibataire et du fils illégitime, tout en augurant du caractère anormalement agressif d'une relation filiale dont le 
psychanalyste S. Kakar s'est plu a rappeler la nature couramment symbiotique en Inde (1978 : chap. III).

En insistant complaisamment sur la bâtardise de Parasurāma, les récits populaires rejoignent la tradition textuelle qui fait descendre Jamadagni d'une lignée brahmane, celle des Bhārgava, constamment associée aux démons (asura). On discerne là le signe d'un désordre latent auquel l'avatāra (Paraśurāma) viendra lui-même porter remède (Biardeau 1969a : 66).

Plus encore. Les rapports équivoques que cette famille entretient avec la classe des Ksatriya font de ses principaux représentants des personnages mixtes : héros martiaux d'une lignée contemplative, tour à tour acteurs ou victimes. Pour être ambivalente, la relation à la violence de ces " renonçants-guerriers " n'en est pas moins permanente (Goldman 1977 : 138 et suiv.). Loin donc de constituer un cas unique, la figure du Brahmane combattant est plutôt une constante de la mythologie sud indienne ${ }^{11}$, comme elle fut d'ailleurs une réalité sociale tangible dans la plaine indo-gangétique (1978).

La version canonique du mythe de Renukā elle-même illustre, sur le mode fort de la matrimonialité, ce mélange (varnasamkara) des castes Brahmane et Ksatriya normalement prohibé dans l'hindouisme. Non seulement les épousailles de Jamadagni entérinent l'impureté originelle et continue de sa lignee, mais elles l'aggravent : il est la progéniture d'un Brahmane, Rcīka, et d'une princesse, Satyavatī; l'enfantement résulta d'un échange malencontreux de gruaux rituels (avec Vișvāmitra) entre mère et grand-mère maternelle (Biardeau 1981, II, 240). Remarquons cependant que ces mélanges contre-nature ne produiront explicitement leurs effets que sur le petit-fils. Seul Paraśurāma, en effet, incarnera la contradiction d'être un Brahmane violent, un Brahmane doté des vertus parfaites du guerrier. C'est sur lui que finalement se cristallise toute l'équivocité de la lignée. Ce qui

11 Ainsi qu'y insiste M. Biardeau (1968; 1969b). Idée que confirme D.D. Shulman dans son enquête sur la royauté médiévale (1985: 111 et suiv.) : le mythe de Paraśurāma n'est pas sans ressemblance avec celui d'Aurva ; ce qui conduit J.A.B. Van Buitenen à avancer que le second permettait de reconstruire de manière convaincante la préhistoire textuelle du premier (1978; 146-150). 
ne signifie pas que Parasurãma soit simplement un héros martial dont les exploits visent la conquête de la souveraineté universelle, contrairement à l'interprétation de R. P. Goldman ${ }^{12}$. S'il devient violent, c'est seulement pour répondre aux ordres paternels. Sa force reste au service exclusif du Brahmane. Il s'agit d'abord de purifier Jamadagni de la souillure provoquée par la sexualité de son épouse, entendons métaphoriquement la Terre devenue impure : les effigies de Yellamma déposées sur le sol - simples pierres éventuellement rougies et pourvues d'yeux globuleux - ne suggèrent-elle pas que son corps est le village tout entier, synecdote de la Terre ? Il s'agit de punir ensuite le prince, normalement destiné à la protection du dharma, qui déséquilibre le monde en commettant le brahmanicide. Si Parasurāma quitte sa méditation himalayenne pour descendre parmi les hommes, c'est essentiellement pour secourir les Brahmanes de l'oppression ksatriya (Biardeau $1968 ; 1970 ; 1976 ; 185$ et suiv.). Parasurāma ne fait que remplir son devoir (svadharma) inscrit dans son ambiguïté lignagère en sanctionnant les transgressions de l'épouse et du roi. Sa force de kṣatriya, aliénée aux Brahmanes auxquels il appartient, a pour effet de détruire l'ancien univers désordonné (adharma). Toutefois ce désordre est proprement sa vocation : de chacune de ses interventions (re)naît l'ordre (dharma), c'est-à-dire ce mode où la complémentarité hiérarchique entre Brahmane et Ksatriya est restaurée.

Abandonnons l'interprétation normative lettrée pour adopter maintenant le point de vue des Intouchables. Pour ceux-ci, Parasurāma incarne sans conteste la figure du bâtard. Certes, il est né du sperme vagabond d'un roi ou de la chevelure miraculeuse d'un ascète. Mais il est d'abord le fils illégitime d'une Intouchable, comme un mythe l'explicite en renouvelant sur le mode de la « cuisson » le motif de la naissance miraculeuse :

12 Plus pertinent semble le lien énoncé par R.P. Goldman (1977) de l'équivoque lignée des Bhārgava avec la thématique de la mort et de la renaissance, surtout lorsqu'on se réfêre aux grandes fêtes annuelles qui se déroulent au temple de Saundatti (cf. Assayag 1992a: part I, chap. V). 
Paraśurāma ne cessait de questionner sa mère afin de connaître l'identité de son père. De guerre lasse, Yellamma finit par lui raconter les circonstances de sa naissance.

Ayant l'habitude de mendier pour subvenir à ses besoins, elle s'arrêta un jour devant la maison d'une servante appelée Mātangi. Mais celle-ci n'avait rien à manger. La mendiante insista. Mătangi ramassa alors quelques grains et lui donna. Yellamma lui demanda de l'eau afin de les faire bouillir, mais Mătangi n'avait pas non plus de récipient. La déesse lui fit verser l'eau dans sa paume qui contenait les graminées et le liquide commença à frissonner. Neuf mois durant l'eau bouillit sans discontinuer. De cette ébullition naquit Paraśurāma.

Selon les Intouchables, Parasurāma est un Intouchable. Son intervention n'a pas tant pour fonction de restaurer l'harmonie des varna que de renouer avec un monde fécond. Son identité de «bâtard de la déesse » estompe sa parenté avec Jamadagni-Śiva. Conséquemment, les membres des castes subalternes tendent à minorer le rôle masculin de l'avatãra pour insister plutôt sur celui, autrement déterminant, de la déesse à la nature féconde. Voilà bien ce qui les intéresse : non pas tant le rétablissement d'un univers hiérarchisé que celui de l'ordre fertile et prospere. Ce pour quoi ils rendent d'abord des cultes à Yellamma afin de retrouver une nature dispensatrice de bienfaits.

Loin d'être contradictoires, les interprétations lettrée et populaire peuvent être complémentaires. Ainsi D.D. Shulman mentionne-t-il un texte tamoul du XVIIe siècle, le Civiñannayokika, dans lequel Parasurāma devient un « brahmane-intouchable " (pärppanakkataiyañ) pour avoir commis le matricide, ce péché suprême (mahāpāpa) qui souille radicalement (1985: 128). A terme, le Brahmane-guerrier, écartelé entre des statuts extrêmes, fait se rejoindre pureté et impureté. Il les transcende plutôt, dans une conjonction où l'on reconnaît la figure du renonçant-ascète, justement situé au-delà du pur et de l'impur. A Saundatti, les dévots des hautes castes aiment dire de Paraśurāma qu'il est perpétuellement et entièrement absorbé dans l'accomplissement du devoir pieux sur l'Himalaya, lorsque ses parents n'en appellent pas à son soutien. Mais cette distance n'empêche nullement les Intouchables de faire de cet héroïque méditant l'un des leurs. 
La préhistoire familiale de Yellamma elle-même aide à comprendre la tragique destinée des divinités du lieu. Si l'on sait qu'en Inde domine la filiation patrilinéaire, on découvre - pour autant qu'on mette de côté le récit canonique (aseptisé) des épousailles de Renukä $\bar{a}^{13}$-, une Yellamma pourvue d'une seule ascendance matrilinéaire sous le nom de Satyamma (ou Satwāi en marāthi), dont le temple se trouve en contrebas de la colline de Saundatti. Recueilli auprès d'une femme de caste Marātha, un récit explique que Satyamma abandonnait chaque nuit sa fille pour aller remplir sa mission divine. Le désespoir de l'enfant était grand. D'autant plus fort qu'elle apprit de la bouche de sa propre mère qu'elle épouserait plus tard son frère :

Horrifiée à l'annonce de cette prédiction, la jeune fille décida de ne jamais se marier. Un jour qu'elle s'abreuvait à une rivière, elle avala sans le savoir le sperme d'un roi qui jouait dans le cours d'eau avec ses courtisanes. Elle s'aperçut bientôt qu'elle était enceinte. Après l'accouchement, elle enveloppa l'enfant dans un de ses sãri et le jeta du haut d'une montagne afin de ne pas devenir incestueuse. Mais le garçon fut miraculeusement sauvé en tombant sur les branches d'un margousier. Recueilli par un couple de jardiniers sans progéniture, il grandit dans cette famille heureuse de voir son désir d'enfant ainsi comblé par les dieux.

De son côté, renonçante, la jeune femme vivait dans la forêt. Mais, au fil des ans, elle se lassa de la solitude et décida de retourner dans le monde.

A la suite d'une longue errance pour trouver son chemin, elle tomba sur une maison appartenant à un couple qui la reçut avec chaleur. Comme elle ne savait où aller, le couple lui proposa de s'installer dans la maisonnée. Pour les remercier de leur hospitalité, la jeune femme travaillait d'arrache-pied. Le couple bénit les dieux d'envoyer une jeune femme si industrieuse après leur avoir déjà fort généreusement donné un garçon. Avec son

13 En voici le récit : La belle Renukā atteignit l'âge de huit ans, ses parents se proposèrent de la marier. Son père, Renukārāja, le roi du Cachemire, envoya un ministre afin de trouver un époux digne de sa fille. Celui-ci visita en vain plusieurs royaumes pour lui trouver un mari acceptable. Inquiets, le roi et la reine consultèrent le * voyant * (rsi) Agastya qui conseilla de lui faire épouser Jamadagni, le seigneur Śiva lui-même. Après avoir obtenu l'approbation du saint (muni) Rucika, le mariage fut célébré en grande pompe. 
accord, il fut décidé qu'elle deviendrait leur belle-fille. Le mariage fut bientôt consommé.

Devenue maîtresse de maison, la jeune femme continuait de s'affairer sans relâche aux travaux domestiques. C'est ainsi qu'un jour elle trouva par hasard dans la demeure le sart qui enveloppait l'enfant qu'elle avait tenté de supprimer. En interrogeant sa bellemère, qui lui raconta l'histoire de son fils adopté, elle comprit que la prédiction de sa mère, Satwā, était réalisée.

Evoquant d'autres récits similaires, I. Karve reconnaît dans ce récit la version, et l'inversion, proprement marāthi de l'histoire d'Oedipe (1950 ; $c f$. également Ramanujan 1983). L'anthropologue nous apprend surtout que le dénouement - qu'il me fut impossible d'obtenir sur place - n'en est nullement tragique : la jeune femme tint l'inceste secret et vécut heureuse avec son fils-époux sous la protection de ses beaux-parents!

\section{Māțangi, Marikambā, Yellamma}

Si l'on emprunte le chemin grimpant, dans l'axe ouest-est du temple de Yellamma, on parvient à celui de Mātangi, sa " grande (mahā) sœur puînée (tangi)" qui lui tourne le dos. De l'intérieur du sombre sanctuaire de Mātangi, on peut apercevoir le temple de Yellamma grâce à une meurtrière creusée dans le mur du fond. On qualifie souvent Mātangi de upasika, "celle qui est assise près de ", formule appliquée également aux serviteurs (däst) des dieux - notamment les fameuses "danseuses " de temple, dites devadāsi (Assayag 1992, part II).

Conformément à la mythologie classique où Mātangi est l'une des dix manifestations (mahāvidyā) de Satī: celle, furieuse, que prit l'épouse de Śiva pour n'avoir pas été invitée au grand sacrifice célébré par son père Dakșa (Kinsley 1986:162), elle est représentée ici en position de danseuse "terrible ", armée d'une épée. Mātangi est la sentinelle de Yellamma, la déesse guerrière qui protège sa grande sœur de l'attaque des démons, m'apprend le püjari de son sanctuaire, de caste Mādiga. 
En dépit d'efforts répétés, je n'ai pu retrouver localement l'intégralité des séquences du mythe rapporté par nombres d'observateurs (Oppert $1893: 466-467$; Thurston $1909: 4$ : 301-302 ; Moffatt 1978 : 248-249 ; Beck $1981: 126$ et suiv.), celui qui explicite la complémentarité de Yellamma et de Mātangi. Mais une bribe narrative esquisse le motif à sa manière :

Ayant eu vent de la menace de Jamadagni, que l'obéissant Paraśurāma ne pouvait qu'accomplir, Yellamma parvint à lui échapper ; mais, dans sa course folle, elle perdit un à un tous ses vêtements. Epuisé, elle trouva refuge dans un village d'Intouchables. Arrivé sur place, Parasurāma furieux massacra la totalité des villageois. Retrouvant finalement sa mère, il la décapita, mais sans s'apercevoir qu'il venait d'effectuer une double décollation : Mātangi se tenait juste derrière sa maîtresse !

Une fois la décollation opérée, l'une des têtes sectionnées s'envola jusqu'à Jamadagni. L'ascète ne reconnut pas le visage de son épouse et demanda qui elle était, et quelles étaient les raisons de sa venue à l'ermitage. Apprenant son identite, il lui ordonna d'aller vivre chez les Intouchables dont elle deviendrait néanmoins la déesse.

Selon d'autres informateurs, c'est Jamadagni lui-même qui, par erreur, remit le chef (haut/pur) de Yellamma sur le tronc (bas/impur) de Mătangi. A la faveur d'un détail, une autre version, qui rappelle le récit de la naissance de Ganeśa (O'Flaherty 1975 : 262-269), inverse la hiérarchie de la " recapitation":

Respectant la promesse faite à son fils, Jamadagni accepta que Yellamma renaisse à la vie. Mais, dans sa colère, Jamadagni avait réduit en cendres la tête décapitée de son épouse. Parasurāma dut se mettre à la recherche d'un autre chef pour sa mère. Pour ce faire, il décida de décapiter la première femme qu'il rencontrerait. Peu de temps après, Mātangi croisait son chemin...

Il arrive aussi que le dénouement de l'histoire differe : Paraśurāma retrouve alors la tête sectionnée de Mātangi qu'il dépose sur le tronc de Yellamma. Une autre variante illustre combien, en dépit des maladresses successives, le crime s'avère presque parfait :

Paraśurāma décapita sans s'en rendre compte deux femmes, Yellamma et Mātangi, au lieu de sa seule mere. Accédant au désir de voir revenir à la vie Yellamma, Jamadagni lui donna de l'eau 
sacrée pour recomposer le corps. Ce qu'il fit tôt le matin alors qu'il faisait encore nuit, et donc se trompa. Il remit la tête de sa mère sur le tronc de la servante, le chef de Mătangi sur le corps de Yellamma.

Lorsque le jour se leva, Parasurāma prit conscience de sa maladroite inversion. Répugnant à commettre un nouveau double meurtre, il décida de présenter à son père les deux femmes en l'état, sollicitant son pardon. Le sage l'accepta, et reprit pour femme celle qui avait la tête de Yellamma. Il fit de l'autre une déesse pour les Intouchables.

Dans la plupart des récits, le sort malheureux de ces deux femmes, liées par une relation ancillaire, reste joint. Un épisode, recueilli à Chandragutti, petite localité célebre pour ses rites de nudité liés à Renukāa, pousse la complémentarité des déesses jusqu'à la symétrie en attribuant un fils à Mātangi. Mais c'est un enfant démoniaque, conformément à l'infériorité statutaire de sa génitrice - l'exact inverse du fils dévoué, d'abord à son père, ici à sa mère, qu'est Paraśurāma. Ce parallélisme filial avec inversion de signe rappelle un thème récurrent de la mythologie dévotionnelle sud-indienne : la sentinelle du dieu se transforme en démon (Shulman $1985: 27-32$ ).

Avant d'exterminer tous les Ksatriya, Paraśurāma laissa sa mère sous la protection de son fidèle « lieutenant » Bīrappa, le fils de Mātangi. Tandis que le premier accomplissait sa mission, ce dernier se transforma en démon (asura).

Harcelant les femmes de l'ermitage er du royaume, il enlevait les vêtements de celles qu'il poursuivait. Inlassablement, Mātangi fournissait des habits de fortune aux victimes de son fils, sous la forme notamment de pagnes confectionnés avec des branchages de margousier (utilisés lors des rituels thérapeutiques à Saundatti).

Une fois sa vengeance accomplie, Parasurāma regagna l'ermitage où il apprit l'inqualifiable conduite de son « lieutenant ". Afïn de faire cesser la terreur que Bīrappa inspirait, et dans le but de rétablir l'ordre, Paraśurāma décida de le tuer.

Cette dépendance des deux déesses se constate semblablement pour la série de récits où, cette fois, Yellamma n'est plus la victime du courroux, mais une puissance débarrassée du lien conjugal. Devenue autonome et active, elle apparaît, si l'on ose dire, extrêmement susceptible. Chaque manquement ou inobservance provoque en effet sa 
colère. Elle utilise pour ce faire Mātangi, prenant alors son apparence, pour tester la dévotion à son endroit, par exemple auprès de fermiers dont elle avait coutume de favoriser les récoltes. Sans vouloir ici développer ces récits ${ }^{14}$, indiquons néanmoins qu'à chaque fois les interventions de Mātangi, commandées par Yellamma, se soldent par une mutilation nasale. Dans le contexte local, c'est un signe d'humiliation empêchant tout mariage plutôt que la métonymie d'une décapitation, voire d'une clitoridectomie, ainsi qu'un psychanalyste le prétend (Kakar 1978 : 99). Mais il arrive aussi que Mātangi serve de médiation, telle une incarnation instrumentalisée de Yellamma. Comme lorsqu'un malafoutier tenta de corriger Mātangi. En accord avec sa maîtresse, celle-ci se déguisa alors en jeune femme séduisante pour lui infliger des maladies. Ainsi est-ce par le truchement d'une forme inférieure que la puissance (sakti) de Yellamma agit sans intervenir personnellement. Manifestation semblable, remarquons-le, à celle de son époux, Jamadagni, qui délègue l'exercice de la violence à son fils, Parasurāma. Cela lui évite d'accroître sa souillure en recourant à la force du guerrier, alors même que son "ardeur» (tãpas), ce feu intérieur produit par le méditant, est capable de brûler à distance ses victimes. Dans les deux cas la violence donne lieu à une dévolution. Pour le dieu, l'exécution des basses auvres criminelles passe par la filiation patrilinéaire. Pour la déesse, elle est le fait d'une de ses émanations inférieures, selon la conception panindienne de la Devt, une et multiple, dotée d'un extraordinaire pouvoir de métamorphose et d'une capacité à se scinder en une myriade de manifestations adaptées aux situations où s'impose son intervention ${ }^{15}$.

Tandis que la violence masculine prend la forme d'avatāra - un dieu «descend" sur la terre pour rétablir l'ordre - la violence féminine s'apparente plutôt à une ascension - une déesse, qui ressemble en tous points à une femme ordinaire, se transforme sous l'emprise de la colère : " Une femme furieuse est une déesse ", affirme

${ }^{14}$ On trouvera les versions détaillées de ces récits dans Assayag (1992a: 97-100).

15 Un article développe ces multiples aspects que revêt la divinité, en fonction notamment des contextes d'énonciation (Assayag, 1992b). 
un proverbe kannada (Ramanujan 1986: 61) ${ }^{16}$. Métamorphose qu'illustrent les récits, recueillis dans la ville de Sirsangi, concernant cette fois une autre déesse répondant au nom de Marīkambā. Ils valent comme mythe étiologique du sacrifice du démon-buffle (Mahișa).

Marīkambā, la fille d'un Brahmane, épousa par erreur un tanneur Mādiga. En le voyant chaque jour accompagné de son fils quitter le domicile pour se rendre au travail, elle pensait qu'il tirait ses revenus de son activité de brahmane. Marīkambā ignorait la véritable origine de caste de son époux. Tandis que son père se livrait à l'activité polluante de tanneur, l'enfant avait coutume de s'amuser avec les chutes de cuir.

Un jour, il en glissa une dans la poche de son vêtement, et rentra à la maison. Voulant nettoyer ses habits, sa mère découvrit la laniere. Comprenant subitement la profession et le statut de son mari, elle fut prise d'une rage terrible. Dans sa colere, elle les chassa ; puis les poursuivit. Epuisés par cette course folle, le père et l'enfant déciderent de se cacher, le premier dans un buffle, le second dans un bouc. Arrivée sur la place, Marīkambā les décapita l'un après l'autre.

Fort semblable au précédent, et très populaire chez les Intouchables, un deuxième récit introduit le personnage de Mătangi en la circonstance devenue mère de famille :

Un homme de caste Hōleya tomba amoureux de la fille d'un Brahmane lettré. Grâce à un déguisement (brahmane), il parvint à la séduire pour, finalement, l'épouser. La jeune mariée fut donc régulierement invitée à déjeuner chez sa belle-mère, Mātangi.

Un jour, une fois le repas terminé, Mātangi adressa à sa belle-fille la question suivante : "Comment as-tu trouvé ces petits gâteaux de langue de bœuf rôtie ? " Consternée autant qu'horrifiée, la jeune femme s'enfuit. Une rapide enquête confirma que son époux était effectivement de caste Intouchable. Prise de colère, la jeune épouse se rua chez Mātangi pour le tuer. Mais celui-ci, parvint à lui échapper. A bout de force cependant, il se cacha dans

${ }^{16}$ Contrairement aux * grands * mythes, les versions folkloriques affectionnent un surnaturel domestique qui ne répugne pas au comique, plus empreint de magie terrestre que de tentation cosmique. Écart qu'une formule de A.K. Ramanujan résume : * Myths, by and large, divinize the human; folktales humanize the divine * (1987; XVIII). 
un buffle. Lorsque sa femme le rejoignit, elle le décapita sur le champ.

A quelques différences de détail près, ces deux récits procèdent d'un même motif. Ils font semblablement apparaître que la décollation résulte, et sanctionne, un inacceptable mélange de varna, bref le pire qui soit imaginable. Le mélange des "classes" (varnasamkara) provoque une telle confusion des castes (jätisamkara) qu'il est source de désordre absolu; la leçon est conforme à l'enseignement du dieu progéniteur de la race humaine et en même temps son premier législateur qu'est Manu. Les deux variantes enseignent aussi que le buffle décapité n'est autre que l'époux de caste impure. Comme si un mariage contre nature révélait celle, véritablement démoniaque, que la bestialité du conjoint aurait dû interdire. Dernière remarque : par la décapitation du mari suborneur, la déesse n'annule pas la souillure conjugale mais se transforme de son propre fait en veuve - statut maudit en Inde que nul ne saurait donc vouloir.

La confrontation de ce récit au mythe canonique de Saundatti, fait découvrir que cette vengeance situe Marīkambā sur le même plan que Yellamma après l'assassinat de son époux. C'est la même violence brahmane, infléchit en ksatra, qui entraîne le veuvage des deux déesses, encore que leurs destinées convergentes dans le veuvage resteraient purement contingentes s'il n'y avait l'épisode de la décapitation essentiel aux deux récits. Mieux, s'attachant à la seule mytho-logique de la déesse (Durgā) tueuse de buffle, Mahișasura, Biardeau relevait à quel point la décollation du démon équivalait symboliquement à une auto-décapitation: «la déesse guerrière, victorieuse du démon-buffle, ne peut triompher de son ennemi que parce qu'elle s'est offerte elle-même en sacrifice au début du combat. Elle serait décapitée si elle ne décapitait pas : on peut dire aussi qu'elle se décapite un peu en décapitant son ennemi qui n'est jamais qu'une partie d'elle-même puisqu'elle est mere de l'Univers " (1981, II : 481-482). Qu'ajouter à cette éloquente exégèse sinon qu'elle justifie et éclaire l'homologie de nos deux mythes : la déesse peut être en effet, soit décapitante (= Marīkambā), soit décapitée (= Yellamma). 
Explicitons. Dans les deux cas, la décollation est le prix à payer lorsque la pureté de l'épouse est souillée. Si, d'un côté, c'est l'épouse qui décapite et dans l'autre l'époux par l'intermédiaire du fils, c'est pour une unique raison : la transgression de la règle imprescriptible de la pureté matrimoniale. La déesse et le dieu ainsi s'accordent à décapiter leur conjoint pour avoir été souillés par lui. Quant au renversement des rôles attribués à l'une et l'autre femmes, il répond à l'inversion initiale du statut des conjoints : Marīkambā (Brahmane) est supérieure à son époux (Intouchable), comme l'est Jamadagni (Brahmane) envers Yellamma (Intouchable). Deux unions évidemment révélatrices d'un déséquilibre initial qui laisse présager du drame à venir. En attirant pareillement l'impureté et en souillant irrémédiablement les lignages, l'erreur de Marīkambā et la faute de Yellamma signent un même désordre qui appelle réparation et mort sacrificielle; ce qu'exprime la violence de la décapitation tout en autorisant la restauration. Du point de vue structural, le sexe inverse des victimes décapitées se révèle de peu d'importance au regard de l'inégalité statutaire et de la hiérarchie des castes que l'union matrimoniale a normalement pour fonction de reconduire. La souillure n'est pas moins grande, que l'on épouse un Intouchable ou que l'on trompe son mari avec un roi. L'ambiguïté identitaire des déesses favorise en somme l'homologie des deux récits: la brâhmane Marīkambā attire nombre d'Intouchables et l'Intouchable Yellamma a une ascendance brâhmane sous le nom de Renukā.

L'inversion se répète à sa manière dans la similitude des épisodes narratifs de la poursuite précédant la décapitation. Dans le cas de Marīkambā, l'époux décide de "s'habiller » dans la peau du buffle pour lui échapper, tandis que la course folle de Yellamma provoque son déshabillage involontaire. Autre retournement, celui du rôle du fils, présent dans les deux mythes aux côtés du père, quoique inversement: victime décapitée lorsqu'il se transforme en bouc, sacrificateur sous la figure de Paraśurāma. 
On peut corroborer la prégnance des motifs de la colere et de la décapitation, dans la culture locale, en faisant appel au très populaire mythe de Cirutonda ${ }^{17}$ :

Un pauvre Brahmane shivaïte très pieux se lamentait depuis des années de n'avoir pas d'enfant. Un jour enfin, il eut un garçon.

Śiva qui désirait le mettre à l'épreuve, descendit le visiter. Déguisé en ascète (samnyäsin), il lui demanda de le nourrir en exigeant qu'on serve un repas a base de chair humaine : celle d'un garçon d'une famille très religieuse ayant un fils unique.

Le Brahmane accepta et partit a la recherche de ce singulier aliment. Mais, en dépit de ses efforts, il ne parvint pas à mettre la main sur un garçon. Rentré bredouille à la maison, le couple se mit à pleurer de ne pouvoir satisfaire le vœu de l'ascète. Quand leur enfant les vit dans ce triste état, il leur demanda ce qui n'allait pas. Ecoutant ses parents lui confier les raisons de leur tristesse, il leur dit de se calmer car il pourrait être la victime exigée par le saint homme. Alors que ses parents hésitaient, il les pressa de s'exécuter rapidement sous peine de voir la colère de l'ascète se déchaîner.

Le saint homme exigea que la tête soit d'abord coupée et déposée devant lui, pendant que le corps serait cuisiné. Lorsque l'épouse servit le repas, il lui demanda soudain qu'on lui présente le fils de la maison! "Comment pourrais-je faire venir mon enfant, j'ai coupé sa tête et fait cuire son corps ? " répondit-elle. Il insista, en lui conseillant de l'appeler en répétant son nom à trois reprises. Ce qu'elle fit pour s'entendre dire aussitôt : "Maman $(a m m a) !$ ", et voir venir à elle le garçon souriant. S'inquiétant de savoir ce qu'était devenu la tête déposée devant l'ascète, elle s'aperçut qu'elle était transformée en noix de coco.

Son repas terminé, reprenant sa forme de Siva, le saint homme déclara qu'il était satisfait de leur dévotion. Il les bénit avant de s'en aller en leur demandant de (re)faire ce rituel chaque année.

${ }^{17} \mathrm{~S}$. Hanchett rapporte une version légèrement différente de ce mythe pour le sud de l'Etat du Karnataka (1988 : 266-267) dont il existe différentes variantes dans les langues vernaculaires du sud de l'Inde ; on se reportera surtout à G.H. Roghair pour ses éclairants commentaires (1982:298-307 et 124-125). S'agissant de la décapitation, c'est à notre connaissance, la figure tamoule de Aravān - dont l'origine remonte au Mahäbhārata sous le nom d'Irāvat (VI, 90 1-79) - qui donne lieu au plus grand déploiement rituel que l'on puisse aujourd'hui observer en Inde, comme le montrent les riches analyses de A. Hiltebeitel (1988 : chap. 15 ; 1989 : chap. 9). 
On ne s'attardera pas sur la dimension cannibalique de ce saint shivaïte - un Jañgama, renonçant Virasaiva dans la version traduite par G.H. Roghair (1982 : 297), ou un Kāpālika "porteur de crâne " (et meurtrier de Brahmane) selon D.N. Lorenzen (1989:232) - pour ne retenir du récit que les permutations qu'il opère sur celui de Yellamma. Ce n'est plus ici l'enfant (Parasurāma) qui décapite la mère (Yellamma) sur ordre paternel, mais exactement l'inverse, la mère et le père qui le démembrent pour répondre à l'injonction du saint. Dans les deux cas, le commandement est le fait d'un ascète. Mais, dans l'histoire de Cirutonda, la décollation ne sanctionne pas la faute commise à l'endroit d'un roi (libidineux). Elle vise à satisfaire le souhait d'un chaste renonçant qui n'est autre que Śiva.

\section{Désir et colère des dieux}

Ainsi les dieux, comme les hommes, n'échappent-ils ni au désir ni à la colère: victimes eux-mêmes d'une radicale transformation lorsque l'un ou l'autre les possède. Mais ces passions calamiteuses ont chez eux des effets particulièrement destructeurs. Tandis que le désir ( $k a \vec{m} a)$, dont le prototype est le désir sexuel (de Yellamma), déclenche la cascade de calamités qui s'abat sur les membres de la famille divine et autres puissances apparentées, la colère, dont pâtissent tour à tour les acteurs, enclenche la série des épisodes de !'épopée. Certes, il revient à la puissance du désir de lancer le cycle mythologique de Saundatti. Mais c'est à la colère qu'il doit d'être perpétuellement relancé.

La colere - krödha en sanscrit, et dont le champ lexical en kannada est si riche : kanalu, kōpa, ugra - est bien l'ingrédient actif de la mythologie, comme l'énonce métaphoriquement le nom de Jamād-agni qui signifie “feu dévorant ${ }^{18}$. Elle est non la manifestation épisodique du caractere, mais le facteur dynamique du récit, non

${ }^{18}$ En réalité, comme il est fréquent, l'étymologie de Jamadagni est un problème non résolu puisqu'on peut aussi le traduire par les expressions * celui qui va vers le feu ", * celui dont les feux sont mobiles * (indications de Ch. Malamoud). 
un attribut accidentel, mais une faculté essentielle. Ainsi est-elle toujours référée à une puissance suprême : Fureur personnifiée ${ }^{19}$.

Cette personnification passionnelle, qui scande le dispositif narratif, est le ressort de la machinerie criminelle. Soit autant de crimes presque parfaits. Car malgré, où à cause de maladresses répétées, chacun des protagonistes est successivement le meurtrier et la victime, aucun d'entre eux n'en sort indemne, et nul meurtre n'arrête l'hécatombe : la Colère les emporte tous. Irritation de Jamadagni devant l'impureté de sa femme, en la voyant guérie, et agression vengeresse quant il châtre ses propres enfants ; furie de Parasurāma décapitant Yellamma, et ivresse belliqueuse lorsqu'il la sauve des démons ou extermine les Ksatriya. Pourtant figure meutrière par excellence, Paraśurāma est lui-même la victime de sa propre colère, comme le rapporte un bref récit (cité par Elmore : $1925: 88$ ) :

Une fois Yellamma décapitée, l'épée de Paraśurāma s'envola dans les airs. Horrifié par l'acte qu'il venait de commettre, celui-ci maudit le bras « matricide ». Immédiatement l'épée redescendit et lui coupa l'avant-bras qui retomba dans la poussière près de la tête de sa mère ${ }^{20}$.

Si l'on ajoute le dépit coléreux du roi avide voyant s'échapper la Vache céleste, et si l'on parachève l'énumération par l'évocation de la somme des maux qu'inflige à ses dévots la Déesse Yellamma, dont la colère est légendaire, on mesure combien l'agir des dieux se raconte de fureur en fureur. Un mythe collecté par Beck (1981: 127) atteste que cette passion impériale peut servir à conquérir la souveraineté :

En répudiant son épouse, Jamadagni lui donna sept perles magiques. Mais prise de colère, Renukā les jeta au visage de son mari, et chaque perle devint une pustule variolique. Jamadagni la

19 Analogue à cette * agitation belliqueuse *, à cette * force " née d'elle-même *, appelée le Courroux dans le Rig-Veda (X 83 et 84 ), dont Malamoud a fait la subtile exégèse par deux fois $(1968,1992)$.

20 Un autre récit, rapporté par le même auteur (1925: 129) à propos du mari suborneur, raconte comment la déesse (brahmane) le décapita et lui plaça un de ses bras dans la bouche. Ce sont deux mythes étiologiques du sacrifice du buffle, aujourd'hui interdit : on disposait la tête décapité de l'animal à terre en lui mettant en travers de la gueule une de ses pattes sectionnées. 
supplia de reprendre ses perles et de le guérir ; ce qu'elle accepta à condition qu'il satisfasse son désir d'aller au paradis (svarga). Le marché fut conclu.

Une fois au paradis, Renukā lança ses perles sur Śiva, Visnu et Brahmā et le dieu de la mort, Yāma ; tous furent atteints de la variole. A leur tour, ils demanderent ce qu'elle souhaiterait en échange de leur guérison. Renukā exigea des trois principaux dieux qu'ils cèdent leurs épées, et de Yàma elle obtint le droit de tuer les gens sur la terre à l'aide de son collier.

Au risque de gloser à l'excès, on peut avancer que c'est justement parce que les dieux cedent à la colère, à ce feu négatif qui les dévore, que les divinités n'atteignent jamais la complétude de leur être, à l'inverse de la plénitude qu'incarnerait la parfaite maîtrise de soi conforme à l'idéal ascétique, dont atteste cet échauffement intérieur, éminemment positif, qui résulte du joug disciplinaire et leur confere des pouvoirs surnaturels. Mais la privation volontaire de la colere et du désir est essentiellement le fait du yogin, le renonçant Brahmane, puisque désir et colère sont des passions qui siéent (dans certaines limites) au roi ${ }^{21}$. Ce qui éclaire l'ambiguïté de Paraśurāma - ultime rejeton d'une lignée équivoque de brahmanes-guerriers - dont la vocation colérique provoque le désordre afin de faire obstacle au plus grand désordre qui menace.

C'est justement parce qu'à l'occasion les dieux échouent à dominer leur sens, en prêtant ainsi le flanc à la souillure - grâce donc au désir et à la colere -, qu'ils se laissent distraire et s'emportent, bref "parcourent toutes les chutes possibles avant d'atteindre à la parfaite maîtrise " (Biardeau 1981, II : 523).

\section{Retour au sanctuaire de Yellamma}

Le calme retrouve, retournons au petit sanctuaire du corps morcelé de Yellamma. Quelques dévotes s'apprêtent à accomplir le

21 Sujet d'une controverse entre deux théoriciens de la politique, Kautilya et Bhāradvāja, que présente Ch. Malamoud (1992: 10-11). 
rituel, dit namaskäramudri. Chacune leur tour, elles s'accroupissent devant le dispositif lithique pour lui adresser des questions. Puis elles tentent de soulever la pierre noire qui correspond à la tête de Yellamma. Quelquesunes s'y reprennent à plusieurs reprises. Certaines y parviennent, d'autres pas ; celle-là, visiblement contrariée, renouvelle l'interrogation sous forme affirmative ou négative, ou les deux successivement, afin de s'assurer de la réponse de la "Mère". Ainsi communique-t-on avec la déesse de Saundatti, qui allège ou alourdit sa tête (décapitée) à son gré - signe qu'elle acquiesce ou refuse ce qu'on lui demande. Lorsque la requête a quelque chance d'être exaucée, les femmes lui adressent un chant de dévotion scandé par l'interjection: «Yellamma, Udho! Ôh !». Et le rythme des vibrations des luths qui les accompagnent va crescendo. La corde unique de ce luth fait, dit-on, entendre le timbre de la voix de Yellamma. Le récit suivant explique l'origine de cet instrument de musique :

Alors qu'un démon dénommé Bethasur(a) faisait régner la terreur sur la terre, Yellamma appela son fils pour sauver l'ermitage de Jamadagni. Il s'ensuivit une terrible bataille de laquelle Parasurāma sortit vainqueur. Non content d'avoir terrassé l'adversaire, dans sa fureur il le décapita, le dépeça, puis l'éviscéra. Il tira les nerf's du cadavre et les noua de façon à fabriquer une corde. Perforant le crâne, il y attacha l'une des extrémités de la corde. En y ajustant un tibia devant servir de manche, il fabriqua le luth (propre aujourd'hui aux dévots péripatétiques de la déesse Yellamma ${ }^{22}$ ).

La colère est manifestement bien le facteur de l'épopée.

Jackie Assayag

Institut français (Pondichery)

et Centre d'Etudes de l'Inde et de l'Asie du Sud (Paris)

22 Cet instrument, métonymie de leur fonction gyrovague, évoque le crâne des errants Kāpālika - emblème de leur identité de * meurtrier de brahmane * ; pensons également au Mahavrātin qui fait vaeu, en guise de pénitence pour le meurtre d'un Brahmane, d'errer pendant douze ans avec un crâne humain lui servant de bol à aumônes (Lorenzen 1972 : 43). 


\section{Références bibliographiques}

Assayag, J.

1990 "Modern deva-dāst in India (Karnataka) ", in Rituals and Beliefs in Modern India, G. Einchinger Ferro-Luzzi éd, Delhi, Manohar, 53-65.

1992 a La colère de la Déesse décapitée. Traditions, cultes et pouvoir dans le Sud de l'Inde. Paris, CNRS Editions.

$1992 b$ * L'Un, le couple et le multiple. Un complexe rituel et son panthéon dans le Sud de l'Inde (Kamataka) *, in Classer les dieux ? Des panthéons en Asie du Sud, V. Bouillier \& G. Toffin éds, Purusärtha, 14 (CEIAS/EHESS), 75-98.

1994 * Semence de sang ou de son ? Une caste de prêtrise sectaire dans le Sud de l'Inde (Karnataka) », in Les Ruses du salut. Religion et politiques dans le monde indien, H. Stern \& M.L. Reiniche éds, Purusārtha, 17 (CEIAS/EHESS), 55-85.

Beck, B.E.F.

1981 * The Goddess and the Demon. A Local South Indian Festival and its wider context ", in Autour de la Déesse hindoue, Purusärtha, M. Biardeau éd, 5, 83-136.

Biardeau, M.

1968 *Etudes de mythologie hindoue (I). I : Cosmogonies puraniques *, Bulletin de l'Ecole française d'Extrême-Orient, 54, 1945.

$1969 a$ * Etudes de mythologie hindoue (II). II : Cosmogonies puraniques * Bulletin de l'Ecole française d'Extrême-Orient, 55, 59-105.

$1969 b$ «La décapitation de Renukā dans le mythe de Paraśurāma *, in Pratidanam, J. C. Heesterman éd, The Hague, Mouton, 563-572.

1970 * The Story of Arjuna Kartavirya without Reconstruction *, Purāna, XII, 2, 286-303.

1976 * Etudes de mythologie hindoue (IV). II : Bhakti et avatāra *, Bulletin de l'Ecole française d'Extrême-Orient, 63, 11-263.

1981 Articles * Terre. Les symboles de la Terre dans la religion de l'Inde * (II, 481-482), * Vasistha/Visvāmitra. La séparation des fonctions royales et sacerdotales * (II, 522-523), in Dictionnaire des Mythologies, Y. Bonnefoy éd, II, Paris, Flammarion.

1989 * Brahmane Meat-Eating Gods *, in Criminal Gods and Demon Devotees, A. Hiltebeitel éd, Albany, State University of New York, 19-33.

Gail, A.

1977 Paraśurâma. Brahmane und Krieger. Untersuchung uber Ursprung und Entwicklung eines Avatära Visnu and Bhakta Śiva in der indischen Literatur. Wiesbaden, Otto Harrassowitz. 
Goldman, R.P.

1977 Gods, Priests and Warriors. the Bhrgu of the Mahăbhărata. New York, Columbia University Press.

Hanchett, S.

1988 Coloured Rice. Symbolic Structure in Hindu Family Festivals. Delhi, Hindustan Publishing Corporation.

Hiltebeitel, A.

1988 The Cult of Draupadi, 1: Mythologies: From Gingee to Kuruksetra. Chicago and London, University of Chicago Press.

1991 The Cult of Draupadi, 2 : On Hindu Ritual and the Goddess. Chicago and London, The University of Chicago Press.

Kakar, S.

1987 The Inner World: A Psycho-analytic Study of Childhood and Society in India, Delhi, Oxford University Press.

Karve, I.

1959 * A Marathi Version of the Oedipus Story *, Man, 99, 71-72.

Kingsley, D.R.

1986 Hindu Goddesses. Visions of the Divine Feminine in the Hindu Religious Tradition. Berkeley, University of California Press.

Laplanche, J. \& Pontalis, J-B.

1967 Vocabulaire de la psychanalyse. Paris, P.U.F.

Lorenzen, D.N.

1972 The Käpālikas and the Kälämukhas. Two Lost Saivaite Sects. New Delhi, Thompson Press.

1978 * Warrior Ascetics in Indian History *, Journal of the American Oriental Society, 98, 1, 61-75.

Malamoud, C.

1969 "Un dieu védique : le Courroux : "manyuh svayambhüh", in Mélange d'indianisme à la mémoire de Louis Renou ", Paris, De Boccard, 493-507.

1984 *Vengeance et sacrifice dans l'Inde brahmanique *, in La Vengeance. Vengeance, pouvoirs et idéologies dans quelques civilisations de l'Antiquité, R. Verdier \& J.P. Poly éds, Paris, Editions Cujas, 36-46.

1992 "La colère et le désir dans le système des passions royales de l'Inde ancienne *, Psychiatrie Française, 1, 8-14.

Moffatt, M.

1979 An Untouchable Community in South India. Structure and Consensus. Princeton, Princeton University. 
O'Flaherty, W.D.

1973 Asceticism and Eroticism in the Mythology of Siva. London, Oxford University Press.

Oppert, G.

1893 The Dravidians or the Original Inhabitants of Bharatavarsa. Westminster, A. Constable (Réimp. New/Delhi/Madras, Asian Educational Services 1988).

Ramanujan, A.K.

1983 * The Indian Oedipus*, in Oedipus. A Folklore Case Book, L. Edmunds \& A. Dundes éds, New York and London, Garland Publishing, Inc. 234-266.

1986 * Two Realms of Kannada Folklore *, in Another Harmony. New Essays on the Folklore of India, S.T. Blackburn \& A.K. Ramanujan éds, Berkeley, University of California Press, 41-75.

Ramanujan, A.K. \& S.T. Blackburn (éds.)

1986 Another Harmony. New Essays on the Folklore of India. Berkeley/Los Angeles/London, University of California Press.

Renou, L.

1956 Hymnes spéculatifs du Véda, traduits du sanscrit et annotées. Paris, Gallimard/Unesco.

Roghair, G.H.

1982 The Epic of Palnādu. A Study and Translation of Palnāti Virula katha, a Telugu Oral Tradition from Andhra-Pradesh. Oxford, Clarendon Press.

Shulman, D.D.

1985 The King and the Clown in South Indian Myth and Poetry. Princeton, Princeton University Press.

1989 * Outcaste, Guardian, and Trikster : Notes on the Myth of Kārttavarāyan *, in Criminal Gods and Demon Devotees, A. Hiltebeitel éd, Albany, State University of New York, 33-68.

Thurston, E. (assisté de K. Rangachari)

1909 Castes and Tribes of Southern India, 7 vol. Madras, Madras Government Press (reprint 1975).

Truffaut, F.

1966 Le cinéma selon Hitchcock. Paris, Robert Laffont.

Van Buitenen, J.A.B.

1978 Mahäbhärata. Translated and Edited, vol. 3, The Book of Viräta. The Book of the Effort, Chicago/London, Chicago University Press. 